\title{
Satisfacción laboral y estilos de liderazgo en instituciones públicas y privadas de educación en Chile
}

\author{
Work Satisfaction and Leadership Styles in Public and Private Education Institutions in Chile \\ Satisfaction au travail et styles de leadership dans des institutions d’éducation publiques et privées au Chili
}

Maria M. Chiang Vega

Profesora Asociada, Departamento de Administración y Auditoria, Facultad de Ciencias Empresariales, Universidad del

Bío-Bío. Concepción, Chile. Doctora en Recursos Humanos, Universidad Pontificia Comillas de Madrid, Madrid, España.

E-mail:mchiang@ubiobio.cl

Nelly M. Gómez Fuentealba

Profesora Asistente, Departamento de Estadística, Facultad de Ciencias, Universidad del Bío - Bío, Concepción, Chile. Magíster en Estadística, Universidad de Concepción, Concepción, Chile.

E-mail:ngomez@ubiobio.cl

Carlos M. Salazar Botello Profesor Asistente, Departamento de Gestión Empresarial, Facultad de Ciencias, Universidad del Bío - Bío, Chillan, Chile. Magíster Desarrollo y Comportamiento Organizacional, Universidad Diego Portales, Santiago, Chile.

E-mail:mauricio@ubiobio.cl

Artículo de investigación científica y tecnológica Según clasificación COLCIENCIAS

Recibido: $13 / 06 / 2013$

Revisado: $14 / 06 / 2014$

Aprobado: 14/10/2014

\section{Resumen}

El objetivo de esta investigación es analizar la relación entre la satisfacción laboral y el estilo de liderazgo en 145 docentes de instituciones de educación pública y privada en Chile. Para medir satisfacción laboral se utilizó el cuestionario de Chiang et al. (2008) y para los estilos de liderazgo un cuestionario basado en la teoría situacional de Hersey y Blanchard (Chiang y Salazar 2011). La fiabilidad de las escalas es variable y en todos los casos adecuada, con tamaño de Alfa de Cronbach entre 0.7 y 0.9. Los valores de satisfacción laboral están alrededor de 4 (escala de 1 a 5), situando a los encuestados en un nivel de "laboralmente satisfechos". No hay resultados que indiquen niveles de satisfacción bajos. Los valores respecto de los estilos de liderazgo instrucción, persuasivo, participación y delegación están entre 3 y 3,5, tanto para la muestra femenina como masculina. Los resultados indican que los cuatro estilos de liderazgo están presentes y con niveles medios. Existen correlaciones estadísticamente significativas, en niveles bajos entre los estilos de liderazgo instrucción, persuasión y participación con todos los factores de la satisfacción laboral. Se destacan las correlaciones positivas entre la satisfacción con la forma en que realiza su trabajo y los estilos de liderazgo persuasión y participación. El estilo de liderazgo delegación no se correlaciona con ninguno de los factores asociados con la satisfacción laboral.

Palabras clave: estilo de liderazgo, satisfacción laboral.

\section{Abstract}

The aim of this research was to analyze the relationship between work satisfaction and leadership style in 145 faculty staff in public and private education institutions in Chile. To measure work satisfaction, the questionnaire by Chiang et al., (2008) was used and for leadership styles, a questionnaire was used based on the situational theory by Hersey and Blanchard (Chiang and Salazar 2011). The reliability of the scales is variable and in all cases adequate, with Cronbach's alpha between 0.7 and 0.9. Values of work satisfaction are around 4 (scale from 1 to 5 ), placing those surveyed at a level as "satisfied with their work". No results are available to indicate low levels of satisfaction. Values regarding instruction, persuasive, participation, and delegation leadership styles range between 3 and 3.5, both for the female and male samples. The results indicate that the four leadership styles are present with medium levels. Statistically significant correlations exist, in low levels, among instruction, persuasion, and participation leadership styles with all the factors of work satisfaction. Positive correlations are highlighted between satisfaction with the way their work is carried out and the persuasion and participation leadership styles. The delegation leadership style does not correlate with any of the factors associated to work satisfaction. 


\section{Résumée}

L'objectif de cette recherche est d'analyser la relation entre la satisfaction au travail et le style de leadership sur 145 enseignants d’institutions d’éducation publique et privée au Chili. Pour mesurer la satisfaction au travail on a utilisé le questionnaire de Chiang et al. (2008) et pour les styles de leadership un questionnaire basé sur la théorie situationnelle de Hersey y Blanchard (Chiang y Salazar 2011). La fiabilité des échelles est variable et adéquate pour tous les cas, avec une taille Alfa de Cronbach entre 0.7 et 0.9. Les valeurs de satisfaction au travail sont autour de 4 (échelle de 1 à 5), classifiant aux personnes interrogées sous un niveau de "satisfaits au travail". Il n'existe pas de résultats qui indiquent des niveaux de satisfaction baisse. Les valeurs correspondant les styles de leadership, instruction, persuasif, participation et délégation sont entre 3 et 3,5 si bien pour l'échantillon masculin que pour le féminin. Les résultats indiquent que les quatre styles de leadership sont présents et avec des niveaux moyens. Il existe des corrélations statistiquement significatives, à des niveaux faibles entre les styles de leadership instruction, persuasion et participation avec tous les facteurs de la satisfaction au travail. On peut remarquer les corrélations positives entre la satisfaction avec la façon de réaliser le travail et les styles de leadership persuasion et participation. Le style de leadership délégation n'a pas de corrélation avec aucun des facteurs associés avec la satisfaction au travail.

Mots clef: Styles de leadership, satisfaction au travail.

\section{Introducción}

Hoy, es comúnmente aceptado la existencia de cambios en el significado del trabajo, la naturaleza del trabajo y en la naturaleza de los propios trabajadores; estos cambios han producido diferentes tipos de problemas para los líderes de las organizaciones; estos problemas son importantes tanto desde el punto de vista económico como del social (Aldape, Pedrozo, Castillo y Moguel, 2011). Lo anterior indica la necesidad de conductas directivas de trabajo diferentes, que establezcan nuevas relaciones internas. Para generar este cambio, es necesaria una forma diferente de ver el comportamiento de los individuos en las organizaciones, una transformación cultural, que permita modificaciones conductuales individuales tendientes a dar cumplimiento a los requerimientos institucionales. Según Benítez y Valles (2003, citado en Küester-Boluda y Avilés-Valenzuela, 2011) se puede considerar que las actitudes, competencias y conocimientos de las personas que trabajan en la organización, influyen directamente en sus resultados, su reputación y en definitiva en su éxito. En este contexto, la efectividad o inefectividad de una organización está en gran medida determinada por la calidad de sus líderes.

Por lo anterior, es de vital importancia conocer los estilos de liderazgo y el nivel de satisfacción de los trabajadores con el que se relacionan. De esta manera el objetivo de esta investigación es analizar la relación exis- tente entre la satisfacción laboral y el estilo de liderazgo, en instituciones de educación privada y pública en Chile.

\section{Revisión de la literatura}

Las disciplinas del comportamiento organizacional contribuyen a comprender la conducta de las personas al interior de la organización y es de mucha ayuda al momento de tratar de predecir o estimar algunos comportamientos en la organización (Robbins, 2004).

Lo anterior implica, de acuerdo con Hersey, Blanchard y Johnson (1998), que se necesitan no solamente habilidades de tipo técnicas, sino además habilidades sociales para resolver los diferentes problemas a nivel organizacional. La mayor parte de estos problemas se encuentran a nivel de las personas y no a nivel de las cosas materiales, por ello, para mejorar la gestión es importante estudiar el comportamiento organizacional.

\subsection{Satisfacción laboral}

La satisfacción laboral es importante en cualquier tipo de trabajo, no sólo en términos del bienestar deseable de las personas dondequiera que trabajen, sino también en términos de productividad y calidad. Para Boada y Tous (1993), la satisfacción laboral, entendida como un factor que determina el grado de bienestar que un individuo experimenta en su trabajo, se está convirtiendo en un problema central para la investigación de la organización y es uno de los ámbitos de la calidad de vida laboral que ha captado mayor interés. Así, en el caso de esta muestra, colegios municipales y privados con aporte del estado, la variable de satisfacción laboral reviste singular importancia desde el ámbito de la calidad de la gestión de los grupos de trabajo que ellos forman al interior de su institución.

Una de las definiciones más clásicas de la satisfacción es la ofrecida por Locke (1976) quien la define como un estado emocional positivo y placentero resultante de la valoración personal que hace el individuo sobre su trabajo y sobre la experiencia adquirida en el mismo. No se trata de una actitud específica, sino de una actitud general resultante de varias actitudes que un trabajador tiene hacia su trabajo y los factores relacionados.

Según Harpaz (1983), no existe una definición unánimemente aceptada sobre el concepto de satisfacción laboral, además indica que las personas que trabajan usualmente desarrollan un conjunto de actitudes que puede ser descrito por el término general de satisfacción laboral. Luego, Price y Muller (1986) identifican la satisfacción laboral con el grado en el que a los individuos les gusta su trabajo. Garmendia y Parra (1993) la asocia con el sentimiento de bienestar derivado de cubrir las necesidades de cierto nivel a través de los resultados (considerados aceptables) obtenidos como recompensa del trabajo realizado y Spector (1997) la define como un 
sentimiento o actitud hacia el trabajo en su conjunto y en sus distintos aspectos.

Según Peiró (1984), la satisfacción laboral es una actitud general resultante de muchas actitudes específicas relacionadas con diversos aspectos del trabajo y de la organización. De este modo, el estudio de la satisfacción laboral se enmarca dentro del estudio de las actitudes hacia el trabajo junto con el compromiso organizacional y la implicación laboral (Peiró et al., 1995). Siguiendo esta misma línea, Schultz (1995, citado en Ramírez y Benítez, 2012), define satisfacción laboral como la actitud de la persona hacia su trabajo (qué es lo que piensa de este, si está contento o no con su empleo) y supone un conjunto de actitudes o sentimientos respecto a uno mismo. Igualmente, Blum y Taylor (1999) concuerdan en que la satisfacción laboral es el resultado de diversas actitudes que poseen los empleados; esas actitudes tienen relación con el trabajo y se refieren a factores específicos como los salarios, la supervisión, la constancia de empleo, las condiciones de trabajo, las oportunidades de ascenso, el reconocimiento de la capacidad, la evaluación justa del trabajo, las relaciones sociales en el empleo, la resolución rápida de los motivos de queja, el tratamiento justo por los patrones y otros similares.

Otra definición importante es la ofrecida por Davis y Newstrom (2007), quienes definen la satisfacción en el trabajo como un conjunto de sentimientos favorables y desfavorables mediante los cuales los empleados perciben su trabajo. La satisfacción laboral es cambiante porque crecen y decrecen los sentimientos satisfactorios a medida que los motivos de logro se van cubriendo, por lo que las intensidades iniciales se complementan a través de las conductas realizadas.

Así, la satisfacción laboral es básicamente, un concepto globalizador con el que se hace referencia a las actitudes de las personas hacia diversos aspectos de su trabajo. Por consiguiente, hablar de satisfacción laboral implica hablar de actitudes. Luego, se puede concluir con Bravo, Peiró y Rodríguez (1996), que la satisfacción laboral es una actitud o conjunto de actitudes desarrolladas por la persona hacia su situación de trabajo, actitudes que pueden ir referidas hacia el trabajo en general o hacia facetas específicas del mismo.

Específicamente en el área de la educación que se desarrolla esta investigación, los autores Ramírez y Benítez (2012) indican que las instituciones educativas buscan los sistemas, métodos y procedimientos que contribuyan a mejorar sus condiciones operativas desde el interior de la misma, por lo que al existir satisfacción laboral en los trabajadores existe también un impacto positivo en la calidad del servicio.

\subsection{Liderazgo}

Hersey y Blanchard (1972) concibieron un modelo de liderazgo que ha ganado muchos seguidores entre los es- pecialistas del desarrollo administrativo. Este modelo llamado teoría de liderazgo situacional es una teoría de contingencias que se enfoca en los seguidores y establece que para que el liderazgo sea eficaz hay que escoger el estilo correcto que depende de la madurez (grado de preparación) de los seguidores (Hersey, Blanchard y Johnson, 2001). Luego Bass (1985), desarrolló un modelo en el que distinguió entre el estilo de liderazgo transformacional, líderes que producen cambios en la escala de valores, actitudes y creencias de sus seguidores a través de su influencia personal y el transaccional caracterizado por el establecimiento de una especie de transacción entre el líder y los miembros de su grupo.

Para Pascual, el liderazgo implica interrelación de las cualidades, habilidades y necesidades del líder, las necesidades y expectativas del grupo y las exigencias o requisitos de la situación; esta interrelación sugiere que ningún estilo de liderazgo sirve perfectamente para todas las situaciones, por lo que el mejor estilo será aquel que sea considerado el más apropiado para una situación en particular. Según Kotter (1988) el liderazgo es el desarrollo de una visión, siendo capaz de conseguir individuos que puedan apoyar determinadas estrategias y delegar poder en los mismos para que haga realidad esa visión. En tanto que para McGregor (1991), el liderazgo es el proceso persuasivo o el modelo mediante el cual un individuo induce a un grupo a perseguir los objetivos del líder o los objetivos compartidos por el líder y sus seguidores. Para Koontz y Weihrich (2004) el liderazgo es la influencia, esto es, el arte 0 proceso de influir en las personas para que se esfuercen voluntaria y entusiastamente en el cumplimiento de las metas grupales. Los líderes contribuyen a que un grupo alcance sus objetivos mediante la máxima aplicación de sus capacidades. No se ponen a la zaga de un grupo para empujar y presionar; se dejan frente al grupo para facilitar el progreso e inspirarlo a cumplir metas organizacionales.

Los autores nombrados concuerdan en que el liderazgo es el proceso de influir en las actividades de una persona o un grupo, en los esfuerzos por alcanzar una meta en cierta situación. Hay liderazgo en cualquier situación en la que alguien trate de influir en el comportamiento de otro individuo o del grupo.

Dadas las definiciones, se puede decir que el proceso de liderazgo está en función del líder, el seguidor y otras variables situacionales. El liderazgo es la relación entre los líderes y sus seguidores. Es un proceso de influencia mutua, el cual genera un esfuerzo colectivo al servicio de los propósitos y valores comunes del líder y de sus seguidores. Cada vez que alguien intenta influir en el comportamiento de otro, aquel es el líder potencial y el sujeto de su influencia es el seguidor en potencia, sin importar si se trata del jefe, un colega, un subordinado, un amigo, un pariente o bien todo un grupo. 


\subsection{Liderazgo y satisfacción laboral}

La relación entre el liderazgo y la satisfacción laboral es uno de los temas que ha despertado un gran interés a nivel de investigación. Los estudios sobre satisfacción en el trabajo y de liderazgo son importantes no solo porque existe una relación entre satisfacción en el trabajo y algunos factores que afectan el éxito económico de una organización, sino también porque actualmente existe una creciente preocupación humanitaria por la clase de experiencias psicológicas que la gente tiene durante sus vidas, especialmente durante su vida laboral (Aldape et al. 2011). El trabajo debería proporcionar, además de su función económica, un sentido de identidad, significado, auto-estima, un sentimiento de auto-valoración, relaciones sociales, respeto de otros y en general, buenas condiciones de trabajo (Aldape et al., 2011).

A partir de la década de 1950, comenzaron a desarrollarse numerosas líneas de investigación cuyo objetivo principal era averiguar cuáles eran las conductas típicas de los líderes y tratar de relacionar dichas conductas con el rendimiento del grupo y la satisfacción de sus integrantes. En todas ellas, la conducta del líder podía resumirse -aunque con distintos nombres según el modelo o la teoría en la que se incluyen-en dos dimensiones o estilos de liderazgo: orientación a la tarea -el líder está preocupado fundamentalmente por conseguir los objetivos grupales- y orientación a la relación -el líder se interesa básicamente por el bienestar y satisfacción de los seguidores (Cuadrado, Molero y Navas, 2003). También se ha encontrado relación entre la conducta de recompensa del líder y la satisfacción laboral (Podsakoff, Todor y Grover, 1984).

El meta-análisis realizado por Judge y Piccolo (2004) recopila estudios sobre la relación entre estilos de liderazgo transformacional y transaccional con satisfacción encontrando correlaciones significativas y moderadamente fuertes. En otro artículo de Judge, Piccolo y Ilies (2004) se observa la correlación existente entre las dimensiones de consideración y estructura con satisfacción del trabajador, siendo todas significativas. Se observa que el liderazgo ejerce un efecto significativo sobre la satisfacción laboral y debe atender a las necesidades del momento y evaluar qué estilo es el más eficaz para esa situación en especial (Cuadra y Veloso, 2007).

Mediciones tales como estilos de liderazgo y de satisfacción en el trabajo guían a los administradores, no solo hacia un mejor entendimiento de las necesidades de la gente, sino también hacia formas más realistas de mejorar la calidad de vida en el trabajo y a resolver varios problemas laborales que se han encontrado tienen relación con la satisfacción en el trabajo y la clase de líderes con que cuenta la empresa (Aldape et al., 2011)

\section{Metodologia}

El objetivo de esta investigación es analizar la relación entre satisfacción laboral y el estilo de liderazgo en docentes de instituciones de educación pública y privada en Chile. El análisis se realiza segmentando por género y tipo de contrato, de tal manera de establecer las correlaciones necesarias para obtener información que permita tomar decisiones. Para lograr el objetivo se desarrolla un estudio empírico transversal, descriptivo y correlacional.

Al analizar los resultados del estudio, es preciso tener en cuenta la población objeto de estudio y el tamaño muestral. Así, el trabajo de campo se ha llevado a cabo en una sola universidad, por lo que se puede considerar de carácter exploratorio. Además, se ha centrado en los profesores desde un punto de vista personal, de autoevaluación, por lo que las respuestas pueden estar afectadas de tendencia (Küester-Boluda y Avilés-Valenzuela, 2011).

\subsection{Descripción de la muestra}

El universo escogido para esta investigación corresponde a un total de 242 docentes de instituciones de educación pública y privada subvencionada. La selección de este campo de estudio obedece a la necesidad de aportar información relevante que pueda favorecer a una mejor gestión de este tipo de instituciones, porque en la actualidad la gestión en las instituciones de educación pública y privada es un parámetro importante para el análisis de la calidad del servicio entregado. Para definir el tamaño de la muestra se trabajó con un $95 \%$ de confianza, se utilizó varianza máxima y un $5 \%$ de error, lo que implicó una muestra aleatoria de 145 docentes, cuya distribución por tipo de colegio, tipo de contrato y género, se presenta en la Tabla 1.

\begin{tabular}{|c|c|c|c|c|c|c|}
\hline \multirow[b]{2}{*}{$\begin{array}{l}\text { Tipo de } \\
\text { contrato }\end{array}$} & \multicolumn{3}{|c|}{ Femenino } & \multicolumn{3}{|c|}{ Masculino } \\
\hline & $\begin{array}{l}\text { Particular } \\
\text { con aporte } \\
\text { del estado }\end{array}$ & Público & Total & $\begin{array}{l}\text { Particular } \\
\text { con aporte } \\
\text { del estado }\end{array}$ & Público & Total \\
\hline $\begin{array}{l}\text { Indefinido } \\
\text { o planta }\end{array}$ & 52 & 22 & 64 & 24 & 16 & 40 \\
\hline $\begin{array}{l}\text { Definido o } \\
\text { a contrata }\end{array}$ & 20 & 7 & 27 & 8 & 6 & 14 \\
\hline $\begin{array}{l}\text { Total } \\
\%\end{array}$ & 62 & & $\begin{array}{r}91 \\
(63 \%)\end{array}$ & 32 & 22 & $\begin{array}{r}54 \\
(37 \%)\end{array}$ \\
\hline & & uente: C & hiang & Gómez y S & alazar (2 & 014). \\
\hline
\end{tabular}

\subsection{Aplicación del instrumento}

Luego de la socialización inicial, los datos se recogieron aplicando un cuestionario de auto-respuesta anónima y se aplicó sin alterar el funcionamiento normal de las actividades cotidianas. 
Parte I: Cuestionario de liderazgo. Para medir los estilos de liderazgo se utilizó un cuestionario de 36 ítems (Tabla 2) desarrollado y validado por Chiang y Salazar (2011) quienes se basaron en la teoría situacional de Hersey y Blanchard. Con este instrumento se busca conocer los estilos de liderazgo predominantes en esta muestra. Los trabajadores responden utilizando un formato de respuesta tipo Likert de cinco puntos (de "Totalmente de acuerdo $=5$ " a "Totalmente en desacuerdo=1"). Las valoraciones de las escalas se obtienen mediante el promedio de los valores de los elementos de cada escala.

b) Parte II: Cuestionario de satisfacción laboral desarrollado y validado por Chiang, Salazar, Huerta y Núñez (2008) y Chiang, Salazar, Martín y Núñez (2011). Con este instrumento se pretende medir el nivel de satisfacción laboral en trabajadores respecto de ocho dimensiones con un total de 42 ítems (Tabla 3). Los trabajadores responden utilizando un formato de respuesta tipo Likert de cinco puntos.

\subsection{Análisis de los datos}

Una vez recolectados los datos fue necesario organizarlos, clasificarlos y resumirlos adecuadamente, de tal manera que se posibilitara un mejor análisis de la información obtenida. Para tabular los datos se utilizó una planilla de cálculo en Excel, luego el paquete estadístico SPSS 13.0. Los análisis realizados, tanto para la satisfacción laboral, como para los estilos de liderazgo, corresponden al cálculo de estadísticos descriptivos y dado el carácter ordinal de los datos, se aplica la prueba no paramétrica $U$ de Mann-Whitney para efecto de comparar por género, tipo de colegio y tipo de contrato.

\section{Resultados y discusión}

\subsection{Análisis de fiabilidad (coeficiente de consistencia interna o indicador de unidimensionalidad)}

Por fiabilidad se entiende lo que indican los coeficientes de consistencia interna, es decir, el coeficiente Alpha de Cronbach. Lo que se quiere comprobar es si cada ítem mide lo mismo y si las respuestas tienden a covariar, es decir, si los sujetos responden de una manera coherente y de esta manera se pueda deducir que los ítems expresan el mismo rasgo. Schmitt (1996) señala que es usual considerar valores de coeficiente de fiabilidad de 0,50 como bajos, entre 0,60 y 0,70 como aceptables, de 0,80 como meritorios y de 0,90 como excelentes.

En las Tablas 2 y 3 se observan las estimaciones de la consistencia interna de las escalas de liderazgo y de satisfacción laboral. La fiabilidad de las escalas es variable y en todos los casos es adecuada; estos resultados están entre 0.7 y 0.9 , es decir las escalas son consistentes.

\begin{tabular}{|c|c|c|c|c|}
\hline \multirow{3}{*}{$\begin{array}{l}\text { Estilos de } \\
\text { liderazgo }\end{array}$} & \multicolumn{4}{|c|}{ Alfa de Cronbach } \\
\hline & \multirow[b]{2}{*}{$\begin{array}{l}\text { Noode } \\
\text { Ítems }\end{array}$} & \multirow[b]{2}{*}{$\begin{array}{c}\text { Chiang y } \\
\text { Salazar } \\
\text { (2011) }\end{array}$} & \multicolumn{2}{|c|}{$\begin{array}{c}\text { Datos actual } \\
\text { investigación colegios }\end{array}$} \\
\hline & & & $\begin{array}{l}\text { Privado con } \\
\text { aporte del } \\
\text { estado }\end{array}$ & Municipal \\
\hline $\begin{array}{l}\text { Instrucción o } \\
\text { directivo }\end{array}$ & 9 & 0,907 & 0,842 & 0,890 \\
\hline $\begin{array}{l}\text { Persuasión o } \\
\text { persuasivo }\end{array}$ & 10 & 0,866 & 0,913 & 0,874 \\
\hline $\begin{array}{l}\text { Participación } \\
\text { o consultivo }\end{array}$ & 9 & 0,887 & 0,906 & 0,882 \\
\hline $\begin{array}{l}\text { Delegación o } \\
\text { delegativo }\end{array}$ & 8 & 0,762 & 0,563 & 0,724 \\
\hline $\begin{array}{l}\text { Cuestionario } \\
\text { completo }\end{array}$ & 36 & & & \\
\hline
\end{tabular}

\subsection{Análisis descriptivo}

\section{Satisfacción laboral}

Respecto a la satisfacción laboral el instrumento entrega ocho factores: satisfacción por el trabajo en general, satisfacción con el ambiente físico, satisfacción con la forma en cómo realiza el trabajo, satisfacción con las oportunidades de desarrollo, satisfacción con la relación con el jefe, satisfacción con la remuneración, satisfacción con la autonomía y satisfacción con el reconocimiento.

La Tabla 4 entrega los estadísticos descriptivos en cada caso. En general, el valor global de satisfacción está alrededor de 4 , situando a los encuestados en un nivel de "laboralmente satisfechos". Por otra parte, un análisis por factor arroja que tanto en la muestra femenina como masculina, titular o contrata, los valores más altos se encuentran en los factores "satisfacción con la capacidad para decidir autónomamente" y "satisfacción con la forma en que realiza su trabajo" con puntuaciones de 4,1 y 3,9 respectivamente. En tanto que, la "satisfacción con la remuneración" alcanza el valor más bajo correspondiendo a 3,5.

En general, los niveles de percepción de la satisfacción laboral en los profesores de la muestra, están sobre la media (3, en una escala que va del 1 al 5). Es decir, no hay resultados que indiquen niveles de satisfacción bajos.

El análisis comparativo mediante la prueba $U$ de Mann-Whitney por género y por tipo de colegio para cada uno de los factores asociados a "satisfacción" arroja diferencias estadísticamente significativas por tipo de colegio en los factores satisfacción con el ambiente físico del trabajo (U Mann-Whitney $=2314, \mathrm{p}=0,0$ $<0,001)$, satisfacción con la forma en que realiza su trabajo (U Mann-Whitney $=2489, p=0,01<0,05$ ), satis- 
facción con las oportunidades de desarrollo (U Mann. Whitney $=2115,5, p=0,0<0,001)$ y satisfacción con la remuneración (U Mann-Whitney $=2197,5, p=0,0<0,001)$. En todos estos casos se observa una satisfacción más alta en la muestra de profesores de colegios particulares con aporte del estado.

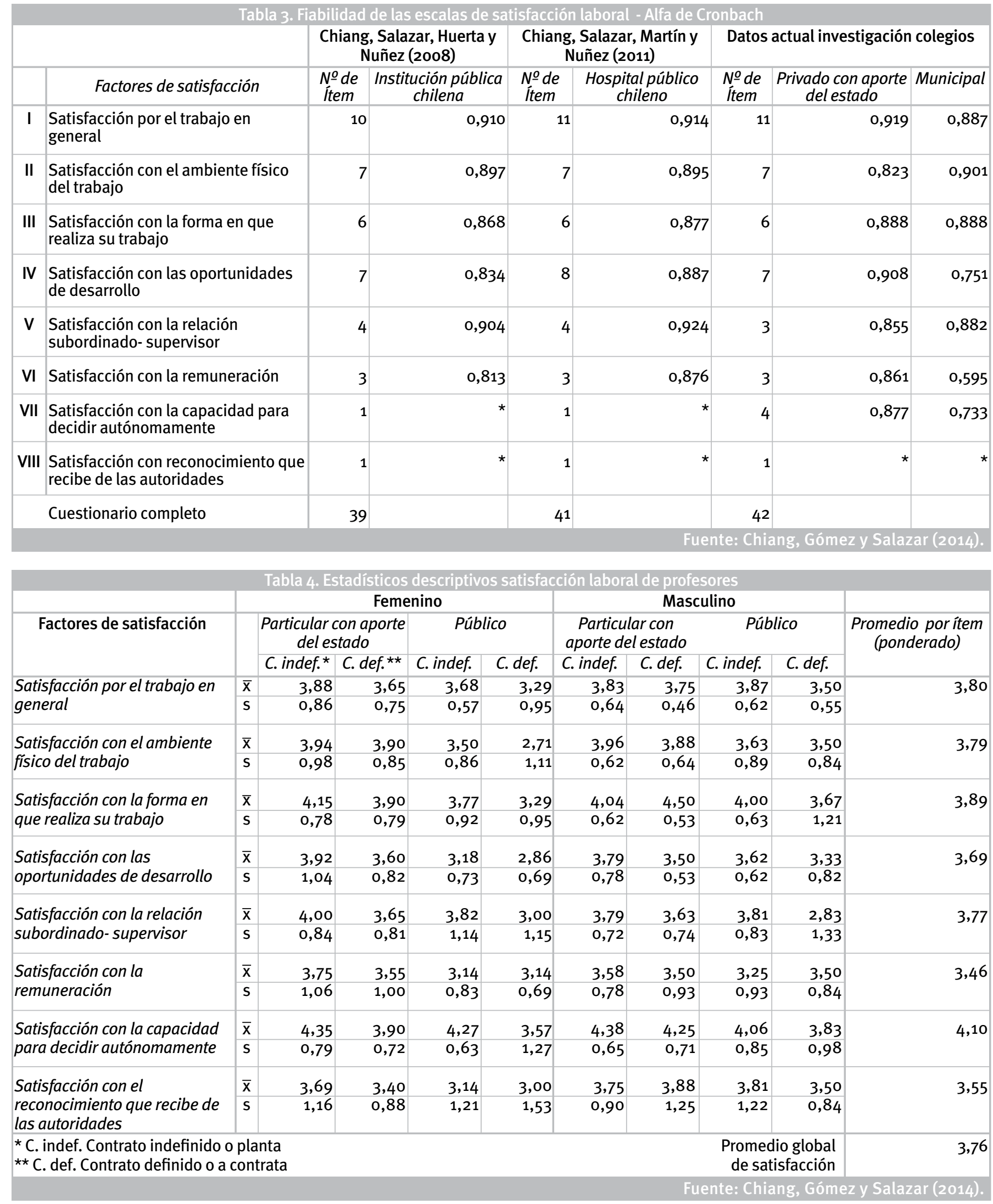




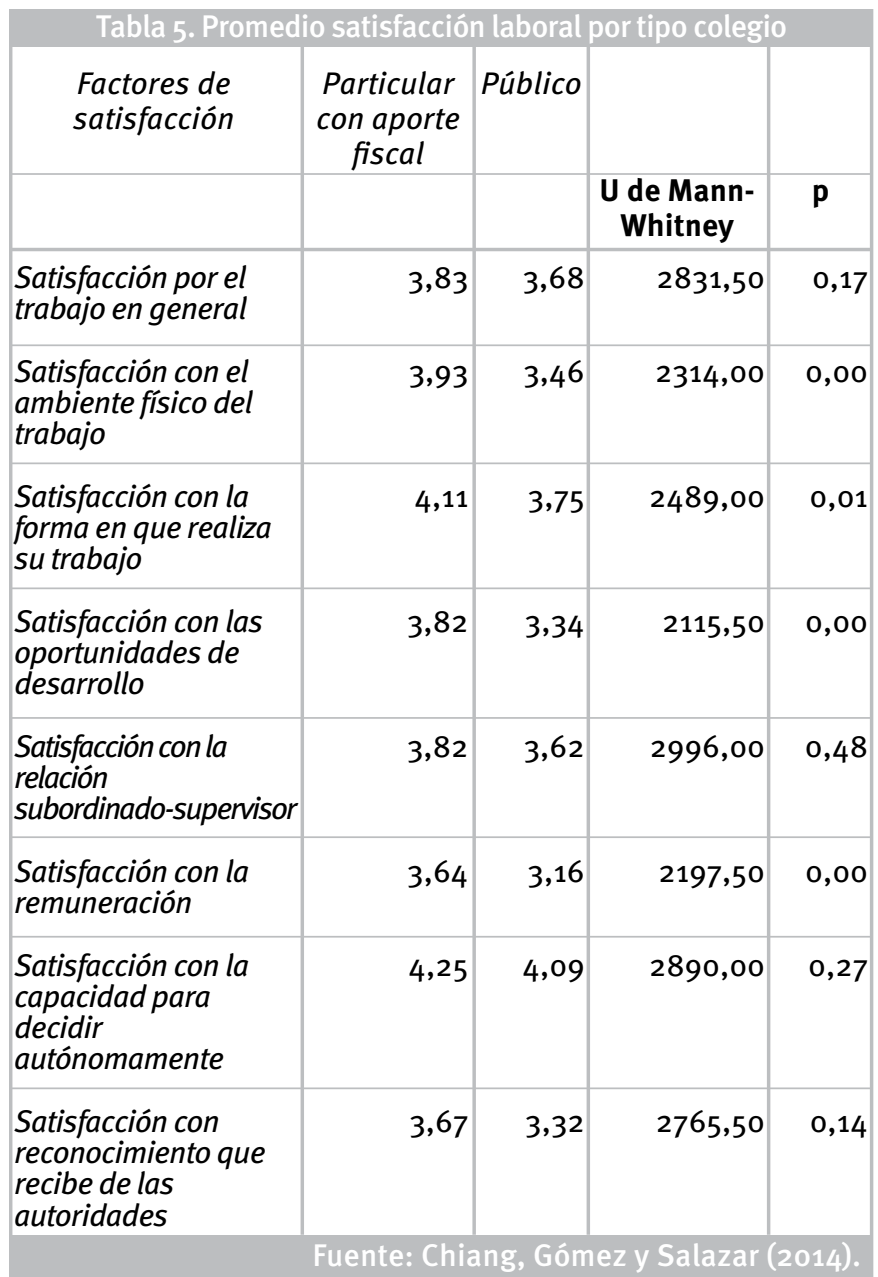

Cuando se compara la percepción por tipo de contrato, se observan diferencias estadísticamente significativas en los factores satisfacción con la relación con el jefe (U Mann-Whitney $=1759,5, p=0,01<0,05)$, satisfacción con la capacidad para decidir autónomamente (U MannWhitney $=1731,5, p=0,01<0,05)$, y satisfacción con las oportunidades de desarrollo (U Mann-Whitney $=1938$, $\mathrm{p}=0,07<0,1)$, destacándose una satisfacción mayor en la muestra de profesores con contrato indefinido. No se encontraron diferencias estadísticamente significativas en ninguno de los factores cuando se hizo la comparación por género.

En la Tabla 6, se observa que los profesores tienen una percepción más alta en cuanto a la satisfacción con la capacidad para decidir autónomamente.

\section{Liderazgo}

Respecto al Liderazgo el instrumento entrega cuatro factores o estilos de liderazgo: instrucción, persuasión, participación y delegación.

La Tabla 7 entrega los estadísticos descriptivos en cada caso. En general, tanto en la muestra femenina como masculina, colegio particular o público, contrato definido o indefinido, los valores respecto del estilo de liderazgo “instrucción" están alrededor de 3,47; liderazgo "persuasivo" alrededor de 3,47; liderazgo "participación" alrededor de 3,45 y liderazgo "delegación” alrededor de 3,02. Estos indicadores muestran que los niveles de percepción del liderazgo en los profesores de la muestra, están sobre la media ( 3 , en una escala que va del 1 al 5). Es decir, los resultados indican niveles altos en los cuatro estilos de liderazgo.

\begin{tabular}{|c|c|c|c|c|}
\hline \multirow[t]{2}{*}{$\begin{array}{l}\text { Factores de } \\
\text { satisfacción }\end{array}$} & $\begin{array}{l}\text { Particular } \\
\text { con aporte } \\
\text { fiscal }\end{array}$ & Público & & \\
\hline & & & $\begin{array}{l}\text { Mann- } \\
\text { Whitney }\end{array}$ & $\mathbf{p}$ \\
\hline $\begin{array}{l}\text { Satisfacción por el } \\
\text { trabajo en general }\end{array}$ & 3,83 & 3,59 & 2009,00 & 0,11 \\
\hline $\begin{array}{l}\text { Satisfacción con el } \\
\text { ambiente físico del } \\
\text { trabajo }\end{array}$ & 3,82 & 3,63 & 2130,50 & 0,33 \\
\hline $\begin{array}{l}\text { Satisfacción con la } \\
\text { forma en que realiza } \\
\text { su trabajo }\end{array}$ & 4,04 & 3,88 & 2159,00 & 0,39 \\
\hline $\begin{array}{l}\text { Satisfacción con las } \\
\text { oportunidades de } \\
\text { desarrollo }\end{array}$ & 3,71 & 3,41 & 1938,00 & 0,07 \\
\hline $\begin{array}{l}\text { Satisfacción con la } \\
\text { relación } \\
\text { subordinado-supenvisor }\end{array}$ & 3,90 & 3,41 & 1759,50 & 0,01 \\
\hline $\begin{array}{l}\text { Satisfacción con la } \\
\text { remuneración }\end{array}$ & 3,51 & 3,46 & 2254,00 & 0,66 \\
\hline $\begin{array}{l}\text { Satisfacción con la } \\
\text { capacidad para } \\
\text { decidir } \\
\text { autónomamente }\end{array}$ & 4,30 & 3,90 & 1731,50 & 0,01 \\
\hline \multirow[t]{2}{*}{$\begin{array}{l}\text { Satisfacción con } \\
\text { reconocimiento que } \\
\text { recibe de las } \\
\text { autoridades }\end{array}$} & 3,63 & 3,44 & 2123,50 & 0,33 \\
\hline & \multicolumn{4}{|c|}{ Fuente: Chiang, Gómez y Salazar (2014). } \\
\hline
\end{tabular}

El análisis comparativo mediante la prueba U de MannWhitney por género, por tipo de colegio y tipo de contrato para cada uno de los factores asociados con "estilos de liderazgo" arroja diferencias estadísticamente significativa por tipo de colegio (Tabla 8) en los cuatro estilos de liderazgo instrucción (U Mann-Whitney $=2103, p=0,0$ $<0,001)$, persuasión ( $U$ Mann-Whitney $=2259, p=0,0$ $<0,001$ ), participación ( $U$ Mann-Whitney $=2170, p=0,0$ $<0,001$ ) y delegación (U Mann-Whitney $=2564, p=0,01$ $<0,05)$. Excepto en el estilo de liderazgo delegación, en todos los otros estilos se observan valores más altos en la muestra de profesores de colegios particulares con aporte del estado.

De la Tabla 8 se puede observar que en promedio los profesores tienen una percepción muy similar entre los estilos de liderazgo directivo, persuasivo, participativo y 


\begin{tabular}{|c|c|c|c|c|c|c|c|c|c|c|}
\hline \multirow[b]{2}{*}{ Estilos de liderzgo } & \multicolumn{5}{|c|}{ Femenino } & \multicolumn{4}{|c|}{ Masculino } & \multirow[b]{2}{*}{$\begin{array}{l}\text { Promedio por ítem } \\
\text { (ponderado) }\end{array}$} \\
\hline & \multicolumn{3}{|c|}{$\begin{array}{l}\text { Particular con aporte } \\
\text { fiscal }\end{array}$} & \multicolumn{2}{|c|}{ Público } & \multicolumn{2}{|c|}{$\begin{array}{l}\text { Particular con } \\
\text { aporte fiscal }\end{array}$} & \multicolumn{2}{|c|}{ Público } & \\
\hline \multirow{2}{*}{$\begin{array}{l}\text { Liderazgo instrucción o } \\
\text { directivo }\end{array}$} & $\overline{\mathrm{x}}$ & 3,77 & 3,50 & 3,09 & 3,00 & 3,50 & 3,88 & 2,94 & 4,33 & \multirow[t]{2}{*}{3,47} \\
\hline & S & 0,61 & 0,76 & 0,75 & 0,58 & 0,51 & 0,64 & 0,77 & 0,82 & \\
\hline \multirow[t]{2}{*}{ Liderazgo participación } & $\mathrm{x}$ & 3,62 & 3,55 & 3,09 & 3,00 & 3,50 & 3,88 & 3,06 & 3,83 & \multirow[t]{2}{*}{3,45} \\
\hline & $\mathrm{s}$ & 0,87 & 0,83 & 0,81 & 0,58 & 0,51 & 0,83 & 0,68 & 0,98 & \\
\hline \multirow[t]{2}{*}{ Liderazgo elegación } & $\bar{x}$ & 3,12 & 2,90 & 3,18 & 3,14 & 2,79 & 3,25 & 3,19 & 4,00 & \multirow[t]{2}{*}{3,02} \\
\hline & $\mathrm{s}$ & 0,51 & 0,55 & 0,50 & 0,38 & 0,41 & 0,71 & 0,54 & 0,63 & \\
\hline
\end{tabular}

de delegación. La prueba U Mann Whitney no arroja diferencias estadísticamente significativas entre profesores con contrato indefinido y definido en la percepción que estos tienen en cuanto a los cuatro estilos de liderazgo estudiados.

\begin{tabular}{|l|r|r|r|r|}
\hline \multicolumn{1}{|c|}{ Tabla 8. Promedio estilos de liderazgo por tipo colegio } \\
\hline $\begin{array}{l}\text { Estilos de } \\
\text { liderazgo }\end{array}$ & $\begin{array}{c}\text { Particular } \\
\text { subvencionado }\end{array}$ & Público & \\
\cline { 2 - 5 } & 3.65 & 3.18 & $\mathbf{2 1 0 3 , 0 0}$ & 0,00 \\
\hline $\begin{array}{l}\text { Liderazgo } \\
\text { instrucción } \\
\text { directivo }\end{array}$ & 3.69 & 3.27 & 2259,00 & 0,00 \\
\hline $\begin{array}{l}\text { Liderazgo } \\
\text { persuasión }\end{array}$ & 3.61 & 3.16 & 2170,00 & 0,00 \\
\hline $\begin{array}{l}\text { Liderazgo } \\
\text { participación }\end{array}$ & 3.01 & 3.27 & 2564,50 & 0,01 \\
\hline $\begin{array}{l}\text { Liderazgo } \\
\text { delegación }\end{array}$ & & Fuente: Chiang, Gómez y Salazar & \\
\hline
\end{tabular}

\subsection{Análisis de correlaciones: Relación entre los estilos de liderazgo y los factores de satisfacción laboral}

En la Tabla 9 se observan las correlaciones (coeficiente de asociación Rho de Spearman) entre las dimensiones de liderazgo y de satisfacción laboral.

Respecto a la relación existente entre las dos variables estudiadas, los resultados muestran correlaciones positivas y estadísticamente significativas, aunque en niveles bajos o moderados entre los estilos de liderazgo instrucción, persuasión y participación con todos los factores de la satisfacción laboral. Destacando correlaciones positivas y estadísticamente significativas entre la satisfacción con la forma en que realiza su trabajo y los estilos de liderazgo persuasión y participación. Esto podría significar que cuando se utiliza estos estilos de liderazgo los trabajadores se sienten más integrados en las decisiones que tiene que ver con la forma en que hacen su trabajo diario, es decir, si los directivos ejercen el liderazgo entregando información que permite convencer y dan posibilidades de participación a los trabajadores, ellos se podrán percibir más comprometidos con la organización.

En lo que se refiere al estilo de liderazgo delegación y tal como se muestra en la Tabla 9, este estilo de liderazgo no se correlaciona con ninguno de los factores asociados con la satisfacción laboral. Esto podría significar que, para esta muestra, un estilo de liderazgo de delegación no aumenta el nivel de satisfacción laboral de los trabajadores de la educación en ninguna de sus dimensiones.

\section{Conclusiones}

El objetivo de esta investigación es analizar la relación existente entre la satisfacción laboral y el estilo de liderazgo, en instituciones de educación privada y pública en Chile y de los resultados obtenidos, se puede concluir lo siguiente:

En la relación existente entre las dos variables estudiadas para esta muestra, los resultados muestran correlaciones estadísticamente significativas, aunque en niveles bajos o moderados, entre los estilos de liderazgo instrucción, persuasión y participación con todos los factores de la satisfacción laboral. En lo que se refiere al estilo de liderazgo delegación no se correlaciona con ninguno de los factores asociados con la satisfacción laboral. Estos resultados, correlaciones estadísticamente significativas, podrían implicar que para esta muestra (profesores de secundaria), los estilos de liderazgo de instrucción, persuasión y participación potencian un aumento de su satisfacción laboral. 


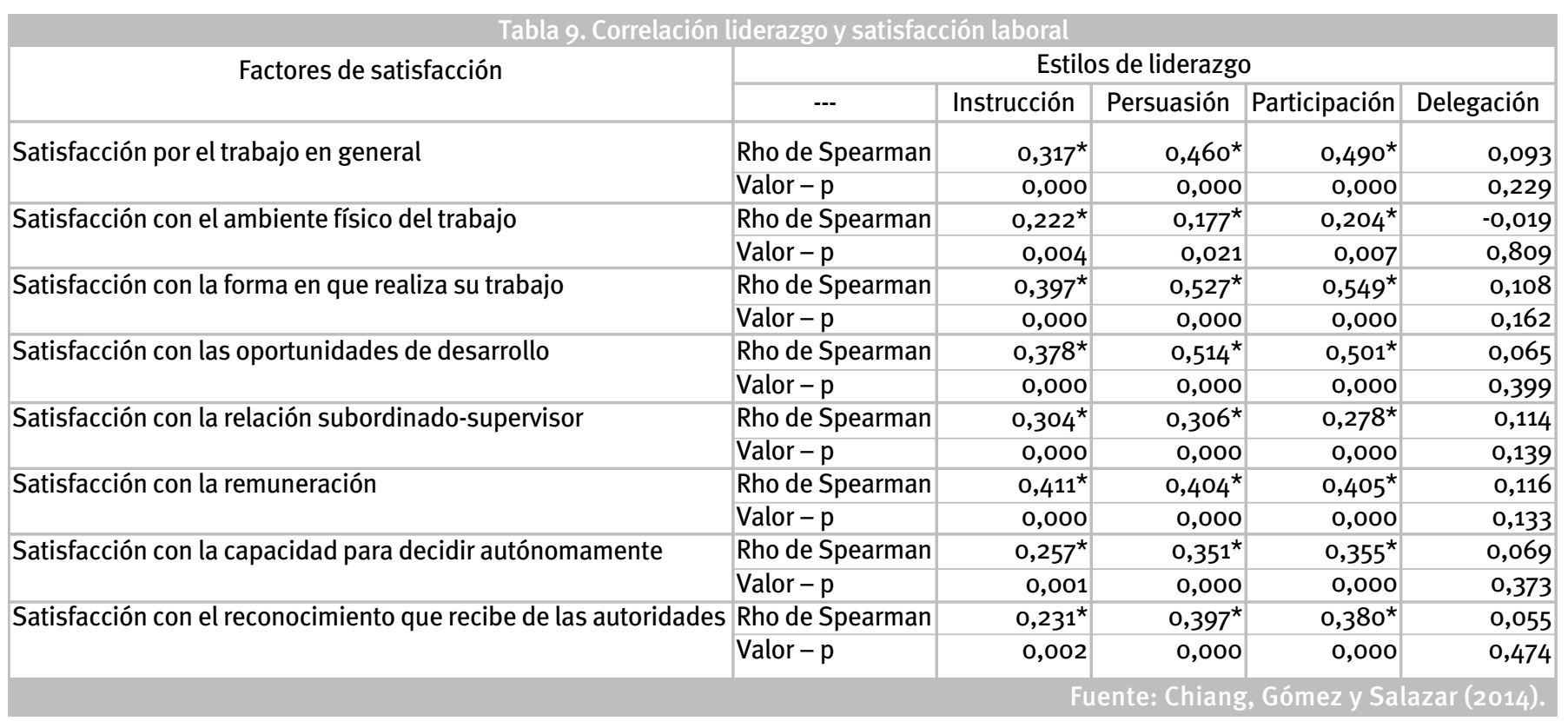

Respecto a los niveles de percepción de la satisfacción laboral en los profesores de la muestra están sobre la media ( 3 , en una escala de 1 a 5), es decir no hay resultados que indiquen niveles de satisfacción bajos. Se observa una satisfacción más alta en la muestra de profesores de colegios particulares con aporte del estado, en los factores satisfacción con el ambiente físico del trabajo, satisfacción con la forma en que realiza su trabajo, satisfacción con las oportunidades de desarrollo y satisfacción con la remuneración. Cuando se compara la percepción por tipo de contrato, se encuentra diferencias estadísticamente significativas en los factores satisfacción con la relación con el jefe, satisfacción con la capacidad para decidir autónomamente y satisfacción con las oportunidades de desarrollo, destacándose una satisfacción mayor en la muestra de profesores con contrato indefinido.

Respecto al liderazgo en general, los valores del estilo de liderazgo instrucción, persuasivo, participación y delegación están entre 3,0 y 3,4. Estos indicadores muestran que los niveles de percepción del liderazgo en los profesores de la muestra están sobre la media (3, en una escala de 1 a 5), es decir, los resultados indican niveles altos en los cuatro estilos de liderazgo. Cuando se compara por tipo de colegio, excepto en el estilo de liderazgo delegación, en los otros estilos se observan valores más altos en la muestra de profesores de colegios particulares.

Sin dudas los hallazgos presentes en este trabajo muestran que a los profesores les interesa mayormente los espacios de participación, esperan un líder que los incite a trabajar colaborativamente donde las relaciones personales son lo más importante, un espacio en el cual se pueda construir un proyecto educativo participativo, que lo lleve a un espacio de satisfacción laboral y al desarrollo personal. Esto concuerda con la visión que se posee de la construcción de un proyecto educativo, el líder educativo debe aglutinar a toda la comunidad en pos de los objetivos que se persiguen. Lo anterior implica que la búsqueda de compromiso y la satisfacción en el lugar de trabajo se manifiesta por las relaciones altamente significativas, donde el líder sea capaz de estimular la participación y persuadir frente a las necesidades educativas y laborales.

Esta investigación puede servir de base para futuros estudios. Por ejemplo, se podría estudiar la direccionalidad de las relaciones entre los estilos de liderazgo y satisfacción laboral aplicando el modelo a muestras similares. Del mismo modo un estudio similar podría realizarse utilizando otras teorías de liderazgo tales como la de rango total (Bass, 1985) con el fin de determinar cómo los estilos de liderazgo afectan la satisfacción laboral y determinar su similitud con los estilos de liderazgo aquí estudiados.

\section{Referencias}

Aldape, A., Pedrozo, J., Castillo, V., y Moguel, M. (2011). Liderazgo y satisfacción en el trabajo ¿Vale la pena estudiarlos? Revista de Economía, Finanzas y Negocios, 1 (2), 35-42.

Bass, B. M. (1985). Leadership and performance beyond expectations. Nueva York, USA: Free Press.

Blum, M., y Taylor, J. (1999). Psicología industrial sus fundamentos teóricos y sociales. México D.F., México: Ed. Trillas.

Boada, J., y Tous, J. (1993). Escalas de satisfacción laboral: Una perspectiva dimensional. Revista de Psicología, 15 (2), 151-166.

Bravo, M., Peiró, J., y Rodríguez, I. (1996). Satisfacción laboral. En Peiró, J., y Prieto, F. (Eds.) Tratado de psicología del trabajo, 1. La actividad laboral en su contexto (pp. 343-394). Madrid, España: Ed. Síntesis S.A.

Cuadra, A., y Veloso, C., (2007). Liderazgo, clima y satisfacción laboral en las organizaciones. Revista Universum de Universidad de Talca, 2 (22), 43-58. 
Cuadrado, I., Molero, F., y Navas, M. S. (2003). El liderazgo de hombres y mujeres: diferencias de género vs diferencias de roles. Encuentros en la Psicología Social, 1 (3), 243-248.

Chiang, M., y Salazar, M. (2011, noviembre). La relación existente entre las creencias organizacionales y el estilo de liderazgo, en una institución pública de educación. Desarrollo y validación de un instrumento. Ponencia ENEFA. Universidad de la Frontera: Temuco, Chile.

Chiang, M., Salazar, M., Huerta, P., y Núñez, A. (2008). Clima organizacional y satisfacción laboral en organizaciones del sector estatal (Instituciones Públicas). Desarrollo, adaptación y validación de instrumentos. Revista Universum, 3 (23), 67-86.

Chiang, M., Salazar, M., Martín M., y Núñez, A. (2011). Clima organizacional y satisfacción laboral, una comparación entre hospitales públicos de alta y baja complejidad. Revista Salud de los Trabajadores, 19 (1), 5-16.

Davis, K., y Newstrom, W. (2007). El comportamiento humano en el trabajo. Comportamiento organizacional. Mexico D.F., México: Mc. Graw- Hill.

Garmendia, J. A., y Parra, F. (1993): Sociología Industrial y de los recursos humanos. Madrid, España: Taurus.

Harpaz, I. (1983). Job satisfaction: Theoretical perspectives and a longitudinal analysis. Nueva York, USA: Libra Publishers.

Hersey, P., y Blanchard, R. (1972). Management of organizational behavior. Nueva Jersey, USA: Prentice Hall.

Hersey, P., Blanchard, K., y Johnson, D. (1998a). Administración del comportamiento organizacional, liderazgo situacional. México D.F., México: Prentice Hall.

Hersey, P., Blanchard, K., y Johnson, D. (2001b). Management of organizational behavior. Lending Human Resources. Upper Saddle River, USA: Prentice Hall.

Judge, T. A., y Piccolo, R. F. (2004). Transformational and transactional leadership: A meta-analytic test of their relative validity. Journal of Applied Psychology, 89 (5), 755-768.

Judge, T. A., Piccolo, R. F., y llies, R. (2004). The forgotten ones? The validity of consideration and initiating structure in leadership research. Journal of Applied Psychology, 89 (1), 36-51.
Koontz, H., y Weihrich, H. (2004). Administración, una perspectiva global. México D.F., México: McGraw Hill.

Kotter, J. P. (1988). The leadership factor. New York, USA: Free Press.

Küester-Boluda, I., y Avilés-Valenzuela, M. E. (2011). El estilo de liderazgo y la orientación al mercado: Su repercusión en la satisfacción en el trabajo del docente universitario. Revista de Globalización, Competitividad y Gobernabilidad, 5 (3), 64-79.

Locke, E. A. (1976). The nature and causes of job satisfaction. In Dunnette, M. D. (Ed.) Handbook of industrial and organizational psychology. Chicago, USA: Rand McNally.

McGregor, J.M., Yu, C.C., Dublin, E. A, Levison, D.A., \& MacDonald, D.M. (1992). Aberrant expression of p53 tumoursuppressor protein in non-melanoma skin cancer. British Journal of Dermatology, 127 (5), 463-469.

Pascual, P. R. (1987). Liderazgo y participación: mitos y realidades. Bilbao, España: Editorial De Deusto.

Peiró, J. M., González-Romá, V., Bravo, M. J., y Zurriaga, R. (1995). La medida de la satisfacción laboral. Ansiedad y Estrés, 1 (2-3), 231-253.

Peiró, J. M. (1984). Psicología de la organización. Madrid, España: UNED

Podsakoff, P. M., Todor, W. D., \& Grover, R. A. (1984). Situational moderators of leader reward and punishment behavior. Fact or fiction? Organizational Behabior and Human Performance, 34 (1), 21-63.

Price, J. L., \& Muller, C. W. (1986): Absenteeism and turnover among Hospital Employees. Greenwich, UK: JAI Press

Ramírez, A., y Benitez, J. (2012). Satisfacción laboral en una institución educativa de nivel superior del Estado de México. Acta Universitaria, 22 (8), 33-42.

Robbins, S. (2004). Comportamiento organizacional. México D.F., México: Eds. Pearson, Prentice Hall.

Spector, P. E. (1997). Job Satisfaction: Application, assessment, causes and consequences. Thousand Oaks, USA: Sage Publications Inc.

Schmitt, N. (1996). Uses and abuses of coefficient alpha. Psychological Assessment, 8 (4), 350-353.

Schultz, P. (1995). Comportamiento organizacional. México D.F., México: Prentice Hall.

Cuadernos de Administración / Facultad de Ciencias de la Administración / Universidad del Valle

Periodicidad: semestral / ISSN impreso Nº120-4645 - ISSN electrónico N²256-5078 / Nombre abreviado: cuad.adm.

Edición Vol. $31 \mathrm{~N}^{\circ} 52$ (julio - diciembre de 2014)

Satisfacción laboral y liderazgo, en instituciones públicas y privadas de educación / Maria Margarita Chiang Vega, Nelly M. Gómez

Fuentealba y Carlos M. Salazar Botello 